NASA Contractor Report 181864

ICASE Report No. $89-43$

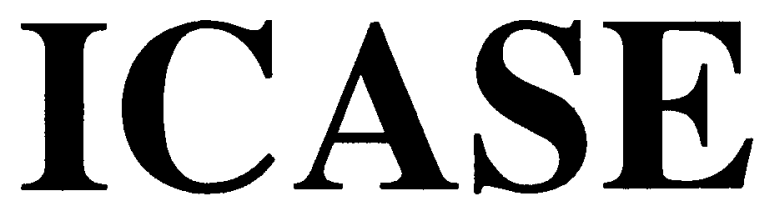

\title{
A CRITICAL COMPARISON OF TURBULENCE MODELS FOR HOMOGENEOUS SHEAR FLOWS IN A ROTATING FRAME
}

Charles G. Speziale

Thomas B. Gatski

N. Mac Giolla Mhuiris

(HASA-CR-181864) A CRITICAL COMPARISOA OF

TURBOLEWCE MODELS FOR HOHOGENEOUS SBEAE

FLORS II A ROTATIYG FRAME Pinal Report

(HaSA. Langley Research Center) $24 \mathrm{p}$ Onclas

CSCL $200 \quad G 3 / 34 \quad 0224106$

Contract No. NAS1-18605

June 1989

Institute for Computer Applications in Science and Engineering

NASA Langley Research Center

Hampton, Virginia 23665-5225

Operated by the Universities Space Research Association

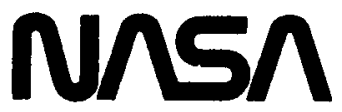

National Aeronautics and

Space Administration

Langley Research Center

Hampton, Virginia 23665-5225 


\title{
A CRITICAL COMPARISON OF TURBULENCE MODELS FOR HOMOGENEOUS SHEAR FLOWS IN A ROTATING FRAME
}

\author{
Charles G. Speziale* \\ ICASE, NASA Langley Research Center \\ Hampton, VA 23665 \\ Thomas B. Gatski \\ NASA Langley Research Center \\ Hampton, VA 23665 \\ Nessan Mac Giolla Mhuiris \\ Case Western Reserve University \\ Cleveland, OH 44106
}

\begin{abstract}
A variety of turbulence models, including five second-order closures and four twoequation models, are tested for the problem of homogeneous turbulent shear flow in a rotating frame. The model predictions for the time evolution of the turbulent kinetic energy and dissipation rate, as well as those for the equilibrium states, are compared with the results of physical and numerical experiments. Most of the two-equation models predict the same results for all rotation rates in which there is an exponential time growth of the turbulent kinetic energy and dissipation rate. The second-order closures are qualitatively superior since, consistent with physical and numerical experiments, they only predict this type of unstable flow for intermediate rotation rates in the range $-0.1 \leq \Omega / S \leq 0.6$. For rotation rates outside this range, there is an exchange of stabilities with a solution whose kinetic energy and dissipation rate decay with time. Although the second-order closures are superior to the two-equation models, there are still problems with the quantitative accuracy of their predictions.
\end{abstract}

* This research was supported by the National Aeronautics and Space Administration under NASA Contract No. NAS1-18605 and was carried out while the author was in residence at ICASE, NASA Langley Research Center, Hampton, VA 23665. 


\section{Introduction}

The problem of homogeneous turbulent shear flow in a rotating frame constitutes a non-trivial test of turbulence models since it incorporates arbitrary combinations of shear and rotation which can have either a stabilizing or destabilizing effect. Since the flow is statistically homogeneous, two-equation turbulence models and second-order closure models give rise to an initial value problem for a coupled set of nonlinear ordinary differential equations. This allows for a fairly detailed mathematical analysis of the models based on dynamical systems theory and for the generation of inexpensive numerical solutions which have a high degree of accuracy. When these features are combined with the wealth of published results from physical and numerical experiments, a powerful tool for the testing and screening of turbulence models is obtained. This establishes the motivation for the present paper which constitutes an extension of the work of Speziale and Mac Giolla Mhuiris (1988).

In this paper, a variety of two-equation models and second-order closure models will be tested for the problem of homogeneous turbulent shear flow in a rotating frame. The models to be considered are as follows:

(i) the standard $K-\varepsilon$ model

(ii) the nonlinear $K-\varepsilon$ model

(iii) the RNG $K-\varepsilon$ model

(iv) the Bardina $K-\varepsilon$ model

(v) the Launder, Reece and Rodi model

(vi) the Rotta-Kolmogorov model

(vii) the Shih-Lumley model

(viii) the Fu, Launder and Tselepidakis model

(ix) the RNG second-order closure model

This represents a broad collection of both two-equation models and second-order closures which includes some of the older models as well as some very recent ones. Comparisons will be made with the physical experiments of Tavoularis and Corrsin (1981) and the large-eddy simulations of Bardina, Ferziger and Reynolds (1983). It will be shown that several of the models yield reasonably good predictions for pure shear. However, the predictions of all of the models degrade considerably for appreciable values of the ratio of the rotation rate to the shear rate, $\Omega / S$. It will be shown that the two-equation models erroneously predict unstable flow (where the turbulent kinetic energy and dissipation rate grow exponentially in time) for all values of $\Omega / S$. The second-order closures will be 
demonstrated to be qualitatively superior to the two-equation models since they predict unstable flow only for an intermediate range of $\Omega / S$; an exchange of stabilities occurs for other values of $\Omega / S$ so that the turbulence decays consistent with the results of physical and numerical experiments. Although the second-order closures are able to account for the stabilizing or destabilizing effect of rotations on shear flow, the quantitative accuracy of their predictions is rather disappointing. The most surprising finding in this regard is that most of the newer models do not yield improved predictions over the older second-order closure models which have a considerably simpler structure.

\section{The Turbulence Models to be Tested}

Three of the two-equation models (i.e., the standard $K-\varepsilon$ model, the RNG $K-\varepsilon$ model, and the Bardina $K-\varepsilon$ model) are of the eddy viscosity type. For these models, the Reynolds stress tensor is of the form (see Hanjalic and Launder 1972)

$$
\tau_{i j}=-\frac{2}{3} K \delta_{i j}+2 C_{\mu} \frac{K^{2}}{\varepsilon} \bar{S}_{i j}
$$

where

$$
\bar{S}_{i j}=\frac{1}{2}\left(\frac{\partial \bar{v}_{i}}{\partial x_{j}}+\frac{\partial \bar{v}_{j}}{\partial x_{i}}\right)
$$

is the mean rate of strain tensor, $\bar{v}_{i}$ is the mean velocity, $K$ is the turbulent kinetic energy, and $\varepsilon$ is the turbulent dissipation rate. The constant $C_{\mu}$ is typically taken to be 0.09 (in the RNG model, $C_{\mu}=0.0837$ ). In the nonlinear $K-\varepsilon$ model of Speziale (1987), a quadratic correction is made to equation (1). For flows in rotating frames, the Reynolds stress tensor for the nonlinear $K-\varepsilon$ model is given by (see Speziale 1989)

$$
\begin{aligned}
\tau_{i j}= & -\frac{2}{3} K \delta_{i j}+2 C_{\mu} \frac{K^{2}}{\varepsilon} S_{i j}-4 C_{D} C_{\mu}^{2} \frac{K^{3}}{\varepsilon^{2}}\left(\stackrel{\circ}{S}_{i j}\right. \\
& \left.+\bar{S}_{i k} \bar{S}_{k j}-\frac{1}{3} \bar{S}_{m n} \bar{S}_{m n} \delta_{i j}+2 \bar{W}_{i k} \bar{S}_{k j}+2 \bar{W}_{j k} \bar{S}_{k i}\right)
\end{aligned}
$$

where $C_{D}=1.68$ and

$$
\begin{aligned}
\stackrel{\circ}{S}_{i j} & =\frac{\partial \bar{S}_{i j}}{\partial t}+\overline{\mathbf{v}} \cdot \nabla \bar{S}_{i j}-\bar{\omega}_{i k} \bar{S}_{k j}-\bar{\omega}_{j k} \bar{S}_{k i} \\
\bar{W}_{i j} & =\bar{\omega}_{i j}+\mathrm{e}_{m j i} \Omega_{m} \\
\bar{\omega}_{i j} & =\frac{1}{2}\left(\frac{\partial \bar{v}_{i}}{\partial x_{j}}-\frac{\partial \bar{v}_{j}}{\partial x_{i}}\right)
\end{aligned}
$$

are the frame-indifferent Jaumann derivative, the intrinsic mean vorticity, and the local mean vorticity. In equation (5), $\Omega_{i}$ is the rotation rate of the reference frame relative to 
an inertial framing and $e_{i j k}$ is the permutation tensor.

The Reynolds stress models (1)-(3) must be solved in conjunction with the transport equation for the turbulent kinetic energy

$$
\dot{K}=\tau_{i j} \frac{\partial \bar{v}_{i}}{\partial x_{j}}-\varepsilon
$$

(which is exact since the turbulence is homogeneous) and a modeled transport equation for the turbulent dissipation rate $\varepsilon$. This modeled transport equation for $\varepsilon$ is of the general form (see Hanjalic and Launder 1972)

$$
\dot{\varepsilon}=C_{\varepsilon 1} \frac{\varepsilon}{K} \tau_{i j} \frac{\partial \bar{v}_{i}}{\partial x_{j}}-C_{\varepsilon 2} \frac{\varepsilon^{2}}{K}
$$

For the standard $K-\varepsilon$ model and the nonlinear $K-\varepsilon$ model,

$$
C_{\varepsilon 1}=1.45, C_{\varepsilon 2}=1.90
$$

whereas for the RNG $K-\varepsilon$ model of Yakhot and Orszag (1986)

$$
C_{\varepsilon 1}=1.063, C_{\varepsilon 2}=1.72
$$

In the Bardina $K-\varepsilon$ model (see Bardina 1988 and Bardina, Ferziger and Reynolds 1983), $C_{\varepsilon 1}$ and $C_{\varepsilon 2}$ are functions of $\bar{W}_{i j}$ given by

$$
\begin{aligned}
& C_{\varepsilon 1}=1.50-0.015 \frac{K}{\varepsilon}\left(\frac{1}{2} \bar{W}_{i j} \bar{W}_{i j}\right)^{1 / 2} \\
& C_{\varepsilon 2}=1.83+0.15 \frac{K}{\varepsilon}\left(\frac{1}{2} \bar{W}_{i j} \bar{W}_{i j}\right)^{1 / 2}
\end{aligned}
$$

The second-order closure models are based on the Reynolds stress transport equation, which for rotating homogeneous turbulent flows takes the form

$$
\dot{\tau}_{i j}=-\tau_{i k} \frac{\partial \bar{v}_{j}}{\partial x_{k}}-\tau_{j k} \frac{\partial \bar{v}_{i}}{\partial x_{k}}-2\left(\tau_{i k} \mathrm{e}_{m k j} \Omega_{m}+\tau_{j k} \mathrm{e}_{m k i} \Omega_{m}\right)-\Pi_{i j}+\varepsilon_{i j}
$$

where $\Pi_{i j}$ and $\varepsilon_{i j}$ are the pressure-strain and dissipation rate correlations, respectively (again, $\mathrm{e}_{i j k}$ is the permutation tensor). In the simplified form of the Launder, Reece and Rodi (1975) model that will be considered in this study, the models for $\Pi_{i j}$ and $\varepsilon_{i j}$ are as follows: 


$$
\begin{aligned}
\Pi_{i j}= & C_{1} \frac{\varepsilon}{K}\left(\tau_{i j}+\frac{2}{3} K \delta_{i j}\right)-C_{2}\left[\tau_{i k}\left(\frac{\partial \bar{v}_{j}}{\partial x_{k}}+\mathrm{e}_{m k j} \Omega_{m}\right)\right. \\
& \left.+\tau_{j k}\left(\frac{\partial \bar{v}_{i}}{\partial x_{k}}+\mathrm{e}_{m k i} \Omega_{m}\right)\right]+\frac{2}{3} C_{2} \tau_{m n} \frac{\partial \bar{v}_{m}}{\partial x_{n}} \delta_{i j} \\
\varepsilon_{i j}= & \frac{2}{3} \varepsilon \delta_{i j}
\end{aligned}
$$

where $C_{1}$ and $C_{2}$ are dimensionless constants that are usually taken to be 1.8 and 0.6 , respectively. Equations (13)-(15) are solved with the modeled dissipation rate equation (8) where $C_{\varepsilon 1}=1.45$ and $C_{\varepsilon 2}=1.90$.

In the Rotta-Kolmogorov model (see Mellor and Herring 1973) and the RNG secondorder closure (Yakhot and Orszag 1988), the models for $\Pi_{i j}$ and $\varepsilon_{i j}$ are given by

$$
\begin{aligned}
\Pi_{i j} & =C_{1} \frac{\varepsilon}{K}\left(\tau_{i j}+\frac{2}{3} K \delta_{i j}\right)+C_{2} K \bar{S}_{i j} \\
\varepsilon_{i j} & =\frac{2}{3} \varepsilon \delta_{i j}
\end{aligned}
$$

where $C_{1}=3.21$ and $C_{2}=0.224$ for the Rotta-Kolmogorov model and $C_{1}=1.59$ and $C_{2}=0.267$ for the RNG second-order closure of Yakhot and Orszag (1988). Both the Rotta-Kolmogorov model and the RNG second-order closure are supplemented by the same modeled transport equation for $\varepsilon$ given in (8); in the Rotta-Kolmogorov model, $C_{\varepsilon 1}=1.8$ and $C_{\varepsilon 2}=2.0$ whereas in the RNG second-order closure, $C_{\varepsilon 1}=1.063$ and $C_{\varepsilon 2}=1.72$. It should be noted that the Launder, Reece and Rodi model, the RNG model, and the Rotta-Kolmogorov model have one characteristic in common: the modeled pressure-strain correlation is linear in the anisotropy tensor

$$
b_{i j}=-\frac{\left(\tau_{i j}+\frac{2}{3} K \delta_{i j}\right)}{2 K}
$$

The Shih-Lumley model (1985) was constructed to satisfy realizability and incorporates more complex nonlinear anisotropic effects. The models for $\Pi_{i j}$ and $\varepsilon_{i j}$ take the form:

$$
\begin{aligned}
\Pi_{i j}= & -\beta \varepsilon b_{i j}+\left(\frac{4}{5}+8 \alpha_{5}\right) K \bar{S}_{i j} \\
& -\frac{2}{3}\left(1-\alpha_{5}\right)\left[\tau_{i k}\left(\frac{\partial \bar{v}_{j}}{\partial x_{k}}+\mathrm{e}_{m k j} \Omega_{m}\right)\right. \\
& \left.+\tau_{j k}\left(\frac{\partial \bar{v}_{i}}{\partial x_{k}}+\mathrm{e}_{m k i} \Omega_{m}\right)-\frac{2}{3} \tau_{m n} \bar{S}_{m n} \delta_{i j}\right]
\end{aligned}
$$




$$
\begin{aligned}
& +\left(\frac{2}{3}+\frac{16}{3} \alpha_{5}\right)\left[\tau_{i k}\left(\frac{\partial \bar{v}_{k}}{\partial x_{j}}+\mathrm{e}_{m j k} \Omega_{m}\right)\right. \\
& \left.+\tau_{j k}\left(\frac{\partial \bar{v}_{k}}{\partial x_{i}}+\mathrm{e}_{m i k} \Omega_{m}\right)-\frac{2}{3} \tau_{m n} \bar{S}_{m n} \delta_{i j}\right] \\
& +\frac{6}{5} \tau_{m n} \bar{S}_{m n} b_{i j}+\frac{4}{15}\left[\tau_{i k} \bar{W}_{j k}+\tau_{j k} \bar{W}_{i k}\right] \\
& +\frac{1}{5 K}\left\{\left[\tau_{i k}\left(\frac{\partial \bar{v}_{j}}{\partial x_{l}}+\mathrm{e}_{m l j} \Omega_{m}\right)\right.\right. \\
& \left.\left.+\tau_{j k}\left(\frac{\partial \bar{v}_{i}}{\partial x_{l}}+\mathrm{e}_{m l i} \Omega_{m}\right)\right] \tau_{k l}-2 \tau_{i l} \tau_{j m} \bar{S}_{l m}\right\} \\
& \varepsilon_{i j}=\frac{2}{3} \varepsilon \delta_{i j}
\end{aligned}
$$

where

$$
\begin{aligned}
\alpha_{5} & =-\frac{1}{10}(1+0.8 \sqrt{F}), \quad F=1+9 \mathrm{II}+27 \mathrm{III} \\
\mathrm{II} & =-\frac{1}{2} b_{i j} b_{i j}, \quad \text { III }=\frac{1}{3} b_{i j} b_{j k} b_{k i} \\
\beta & =2+\frac{1}{9} F\{80.1 \ln [1+62.4(-\mathrm{II}+2.3 \mathrm{III})]\}
\end{aligned}
$$

The turbulent dissipation rate $\varepsilon$ is obtained from the modeled transport equation (8) with $C_{\varepsilon 1}$ and $C_{\varepsilon 2}$ taken as

$$
C_{\varepsilon 1}=1.44, C_{\varepsilon 2}=\frac{7}{5}+0.49[1-0.33 \ln (1-55 \mathrm{II})]
$$

The Fu, Launder and Tselepidakis model (1987) is one of the newest models to be developed by Launder and his coworkers at UMIST. This model satisfies realizability and in the limit of small anisotropies reduces to the simplified version of the Launder, Reece and Rodi model discussed earlier. Its representation for $\Pi_{i j}$ and $\varepsilon_{i j}$ is as follows:

$$
\begin{aligned}
\Pi_{i j}= & -C_{1}^{*} \varepsilon \sqrt{F}\left[2 b_{i j}+4 \gamma\left(b_{i k} b_{k j}-\frac{1}{3} b_{m n} b_{m n} \delta_{i j}\right)\right] \\
& -0.6\left[\tau_{i k}\left(\frac{\partial \bar{v}_{j}}{\partial x_{k}}+\mathrm{e}_{m k j} \Omega_{m}\right)\right. \\
& \left.+\tau_{j k}\left(\frac{\partial \bar{v}_{i}}{\partial x_{k}}+\mathrm{e}_{m k i} \Omega_{m}\right)-\frac{2}{3} \tau_{m n} \frac{\partial \bar{v}_{m}}{\partial x_{n}} \delta_{i j}\right] \\
& +1.2 \tau_{m n} \frac{\partial \bar{v}_{m}}{\partial x_{n}} b_{i j}-\frac{1}{5 K}\left\{2 \tau_{k j} \tau_{l i} \bar{S}_{k l}\right. \\
& \left.-\tau_{l k}\left[\tau_{i k}\left(\frac{\partial \bar{v}_{j}}{\partial x_{l}}+\mathrm{e}_{m l j} \Omega_{m}\right)+\tau_{j k}\left(\frac{\partial \bar{v}_{i}}{\partial x_{l}}+\mathrm{e}_{m l i} \Omega_{m}\right)\right]\right\}
\end{aligned}
$$




$$
\begin{aligned}
& +r\left\{16 \mathrm{II}\left(\tau_{i k} \bar{W}_{j k}+\tau_{j k} \bar{W}_{i k}\right)\right. \\
& \left.-24 b_{m i} b_{n j}\left(\tau_{m k} \bar{W}_{n k}+\tau_{n k} \bar{W}_{m k}\right)\right\} \\
& \varepsilon_{i j}=\frac{2}{3} \varepsilon \sqrt{F} \delta_{i j}-(1-\sqrt{F}) \frac{\varepsilon}{K} \tau_{i j}
\end{aligned}
$$

where

$$
C_{1}^{*}=-60 \mathrm{II}, \gamma=0.6, r=0.7
$$

The dissipation rate $\varepsilon$ is obtained from the standard modeled dissipation rate equation (8) with $C_{\varepsilon 1}=1.45$ and $C_{\varepsilon 2}=1.90$.

For homogeneous turbulent shear flow in a rotating frame (see figure 1), the mean velocity gradient tensor and the rotation rate of the reference frame are given by

$$
\frac{\partial \bar{v}_{i}}{\partial x_{j}}=\left(\begin{array}{ccc}
0 & S & 0 \\
0 & 0 & 0 \\
0 & 0 & 0
\end{array}\right), \Omega_{i}=(0,0, \Omega)
$$

respectively. The nonlinear ordinary differential equations associated with each model are obtained in a straightforward fashion by substituting (28) into the tensor forms given above. These equations were solved using a Runge-Kutta-Fehlburg numerical integration scheme subject to the initial conditions

$$
K=K_{o}, \varepsilon=\varepsilon_{o}, \tau_{i j}=-\frac{2}{3} K_{o} \delta_{i j}
$$

at time $t=0$, which corresponds to an initially isotropic turbulence. Since the transport equations for $\tau_{i j}$ and $\varepsilon$ can be nondimensionalized into an equivalent set of transport equations for $b_{i j}$ and $\varepsilon / S K$, it follows that the solutions will only depend on the initial conditions through the dimensionless parameter $\varepsilon_{o} / S K_{o}$ (see Speziale and Mac Giolla Mhuiris 1988).

\section{Discussion of the Results}

The results obtained for the two-equation models will be discussed first. In figure 2, the time evolution of the turbulent kinetic energy $K^{*} \equiv K / K_{0}$ is shown as a function of dimensionless time $t^{*} \equiv S t$ for three rotation rates: $\Omega / S=0, \Omega / S=0.25$, and $\Omega / S=0.5$. The predictions of the standard and nonlinear $K-\varepsilon$ model, the Bardina $K-\varepsilon$ model, and the RNG $K-\varepsilon$ model are compared with the results obtained from the large-eddy simulations of Bardina, Ferziger and Reynolds (1983) for an initial condition of $\varepsilon_{o} / S K_{o}=0.296$. It is rather disturbing to note that the newer models (i.e., the RNG and Bardina $K-\varepsilon$ models) yield substantially worse predictions than the standard $K-\varepsilon$ 
model for pure shear as shown in figure 2a. While the RNG $K-\varepsilon$ model does well for the case where $\Omega / S=0.25$ (see figure $2 \mathrm{~b}$ ), it appears that this is no more than a coincidence since the model erroneously predicts the same results for all rotation rates and considerably overpredicts the growth rate of the turbulent kinetic energy for the $\Omega / S=0$ and $\Omega / S=0.5$ cases (see figures $2 \mathrm{a}$ and $2 \mathrm{c}$ ). The Bardina $K-\varepsilon$ model does yield results that depend on $\Omega / S$; however, the trends are in conflict with the large-eddy simulations. It predicts that the most energetic case occurs at $\Omega / S=0$ whereas largeeddy simulations indicate that the $\Omega / S=0.25$ case is the most energetic. Furthermore, in contrast to physical and numerical experiments, the Bardina $K-\varepsilon$ model does not predict a flow restabilization (where $K$ and $\varepsilon \rightarrow 0$ as $t \rightarrow \infty$ ) for all values of $\Omega / S$ that are sufficiently far outside of the range $0 \leq \Omega / S \leq 0.5$.

The equilibrium states of each of the two-equation models are compared in Table 1 with the physical experiments of Tavoularis and Corrsin (1981) and the large-eddy simulations of Bardina, Ferziger and Reynolds (1983). It should be noted that since the LES results were not defiltered, they tend to considerably overpredict the anisotropy tensor and should only be considered for the trends that they exhibit. All the equilibrium states were computed with $C_{\mu} \approx 0.09$. Even though improved results are obtained when $C_{\mu}$ is considered to be a function of the ratio of production to dissipation as suggested by Rodi (1972) (which yields $\left(C_{\mu}\right)_{\infty}=0.055$ as used by Speziale and Mac Giolla Mhuiris 1988), in this study we will only show the equilibrium results corresponding to $C_{\mu}=0.09$, since this value is almost always adopted in applications. Several observations concerning these results are noteworthy: (a) both the standard and nonlinear $K-\varepsilon$ models yield the same values for $(S K / \varepsilon)_{\infty}$ and $\left(b_{12}\right)_{\infty}$ which are barely in the range of the physical experiments for pure shear, (b) only the nonlinear $K-\varepsilon$ model is able to reproduce the trends of the large-eddy simulations for the normal components $\left(b_{11}\right)_{\infty}$ and $\left(b_{22}\right)_{\infty}$, and (c) the RNG model drastically overpredicts the values of $\left(b_{12}\right)_{\infty}$ and $(S K / \varepsilon)_{\infty}$ in pure shear which accounts for the overly large growth rate of the turbulent kinetic energy shown in figure $2 \mathrm{a}$.

The equilibrium diagrams of $(\varepsilon / S K)_{\infty}$ are shown as a function of $\Omega / S$ in figure 3 for the standard, nonlinear, RNG and Bardina $K-\varepsilon$ models. It is clear from this figure that all of the two-equation models considered in this study are incapable of properly accounting for the effects of rotations on turbulent shear flow. The standard, nonlinear, and RNG $K-\varepsilon$ models erroneously predict the same results for $K$ and $\varepsilon$ independent of the rotation rate. While the Bardina $K-\varepsilon$ model yields equilibrium states that depend on $\Omega / S$, they appear to be unphysical. For example, there are no stable equilibrium 
solutions for rotation rates outside of the range $-0.9 \leq \Omega / S \leq 1.9$; thus, the model does not predict the flow restabilization that is known to occur for most $\Omega / S$ outside of the range $0 \leq \Omega / S \leq 0.5$.

The time evolution of the turbulent kinetic energy predicted by the various secondorder closure models is shown in figure 4 for the same three cases discussed above. Three important conclusions can be drawn from these results: (a) the Shih-Lumley model and Rotta-Kolmogorov model predict too small a growth rate for pure shear whereas that predicted by the RNG model is too large in the initial stages (see figure 4a), (b) all of the models yield rather bad predictions for the most energetic case of $\Omega / S=0.25$ (see figure $4 \mathrm{~b}$ ), and (c) the Launder, Reece and Rodi model, the Shih-Lumley model and the $\mathrm{Fu}$, Launder and Tselepidakis model erroneously predict stable flow at $\Omega / S=0.5$ whereas the RNG second-order closure has much too strong a growth rate (see figure 4c).

The equilibrium values predicted by each second-order closure is compared in Table 2 with the physical experiments of Tavoularis and Corrsin (1981) and the large-eddy simulations of Bardina, Ferziger and Reynolds (1983). The performance of these models can be summarized as follows: (a) the Launder, Reece and Rodi model does reasonably well in predicting the equilibrium values for pure shear but is defective in its prediction of a premature restabilization at $\Omega / S \approx 0.39$, (b) the Rotta-Kolmogorov model erroneously predicts the opposite extreme wherein the $\Omega / S=0$ and $\Omega / S=0.5$ case are equally energetic since they have the same values for $\left(b_{12}\right)_{\infty}$ and $(S K / \varepsilon)_{\infty}$, and (c) the Fu, Launder and Tselepidakis model does the best in its prediction of the equilibrium states for pure shear; however, it also erroneously predicts a premature flow restabilization at $\Omega / S \approx 0.39$. It should be noted that the erroneous prediction of $\left(b_{12}\right)_{\infty}=0$ and $(S K / \varepsilon)_{\infty}=\infty$ made by the RNG second-order closure is directly tied to its rather low value of $C_{\varepsilon 1}=1.063$. This model could become more competitive with the other secondorder closures if $C_{\varepsilon 1}$ was of the order of $30 \%$ larger.

A characteristic equilibrium diagram for the second-order closures is shown in figure 5 (note that all the models possess the same topological structure for $(\varepsilon / S K)_{\infty}$ taken as a function of $\Omega / S)$. The models have two equilibrium solutions: one where $(\varepsilon / S K)_{\infty} \neq 0$ (the elliptic region $\mathrm{AB}$ ) for which $\varepsilon$ and $K$ grow exponentially with time at the same rate, and one where $(\varepsilon / S K)_{\infty}=0$ for which $\varepsilon$ and $K$ predominantly decay with time. Hence, the second-order closures properly predict unstable flow only for intermediate rotation rates which, depending on the model, can lie anywhere in the ranges $-0.11 \leq \Omega / S \leq 0.39$ to $-0.09 \leq \Omega / S \leq 0.61$. In this intermediate band of $\Omega / S$, the two solutions undergo an exchange of stabilities which qualitatively mimics the shear instability. Furthermore, 
the second-order closures predict a universal equilibrium (i.e., one that attracts all initial conditions) in the unstable regime consistent with the experiments of Tavoularis and Corrsin (1981).

\section{Concluding Remarks}

It has been demonstrated in this study that the existing two-equation turbulence models of the $K-\varepsilon$ type do not properly account for the stabilizing or destabilizing effect of rotations on turbulent shear flow. Furthermore, the quantitative predictions of many of these two-equation models are rather poor for the simple case of pure shear flow. It appears that the nonlinear $K-\varepsilon$ model yields the best results among the two-equation models, however it is still highly deficient. The second-order closures are far superior in a qualitative sense since they do properly predict that there is unstable flow only for intermediate rotation rates that are not too far removed from the unstable regime of $0 \leq \Omega / S \leq 0.5$ predicted by linear stability theory. However, two major problems remain with the quantitative accuracy of the results of the second-order closures: (a) the prediction of a premature flow restabilization at $\Omega / S \approx 0.39$, or (b) the prediction of poor equilibrium values for the case of pure shear, $\Omega / S=0$. Since it is important to predict simple shear flows accurately in engineering applications, it is our opinion that (of the models considered in this study) the Fu, Launder and Tselepidakis (1987) model is on balance the best. However, like the other existing second-order closures, it does not yield accurate results when there is both shear and rotation. The development of significantly improved models based on invariance arguments and a dynamical systems approach will be the subject of a future paper.

\section{Acknowledgment}

One of the authors (MGM) acknowledges the support of the National Aeronautics and Space Administration while in residence at ICOMP, NASA Lewis Research Center, Cleveland, $\mathrm{OH}$. 


\section{References}

Bardina, J. 1988 Turbulence modeling based on direct simulation of the Navier-Stokes equations. Proceedings of the 1st National Fluid Dynamics Congress, Paper No. 883747-CP, 539.

Bardina, J., Ferziger, J. H., and Reynolds, W. C. 1983 Improved turbulence models based on large-eddy simulation of homogeneous, incompressible turbulent flows. Stanford University Technical Report TF-19.

Fu, S., Launder, B. E., and Tselepidakis, D. P. 1987 Accommodating the effects of high strain rates in modelling the pressure-strain correlation. UMIST Mechanical Engineering Department Report TFD/87/5.

Hanjalic, K. and Launder, B. E. 1972 A Reynolds stress model of turbulence and its application to thin shear flows. J. Fluid Mech. 52, 609.

Launder, B. E., Reece, G., and Rodi, W. 1975 Progress in the development of a Reynolds stress turbulence closure. J. Fluid Mech. 68, 537.

Mellor, G. L., and Herring, H. J. 1973 A survey of mean turbulent field closure models. AIAA J. 11, 590.

Rodi, W. 1972 The prediction of free turbulent boundary layers by use of a two-equation model of turbulence. Ph.D. Thesis, University of London.

Shih, T-H., and Lumley, J. L. 1985 Modeling of pressure correlation terms in Reynolds stress and scalar flux equations. Technical Report No. FDA-85-3, Cornell University.

Speziale, C. G. 1987 On nonlinear $K-l$ and $K-\varepsilon$ models of turbulence. J. Fluid Mech. $178,459$.

Speziale, C. G. 1989 Turbulence modeling in non-inertial frames of reference. Theoret. Comput. Fluid Dynamics, 1, 3.

Speziale, C. G., and Mac Giolla Mhuiris, N. 1988 On the prediction of equilibrium states in homogeneous turbulence. Proceedings of the 11th Symposium on Turbulence, University of Missouri-Rolla, B17:1.

Tavoularis, S. and Corrsin, S. 1981 Experiments in nearly homogeneous turbulent shear flows with a uniform mean temperature gradient. Part I. J. Fluid Mech. 104, 311.

Yakhot, V. and Orszag, S. A. 1986 Renormalization group analysis of turbulence. I. Basic theory. J. Sci. Comp. 1, 3.

Yakhot, V. and Orszag, S. A. 1988 Private communication. 


\begin{tabular}{|c|c|c|c|c|c|c|c|}
\hline & $\begin{array}{c}\text { Equilibrium } \\
\text { Values }\end{array}$ & $\begin{array}{c}\text { Standard } \\
\mathrm{K}-\varepsilon \text { Model }\end{array}$ & $\begin{array}{c}\text { Nonlinear } \\
\mathrm{K}-\epsilon \text { Model }\end{array}$ & $\begin{array}{c}\text { RNG } \\
\mathrm{K}-\varepsilon \text { Model }\end{array}$ & $\begin{array}{c}\text { Bardina } \\
\mathrm{K}-\varepsilon \text { Model }\end{array}$ & Experiments & $\begin{array}{c}\text { Large-Eddy } \\
\text { Simulations }\end{array}$ \\
\hline \multirow{5}{*}{$\Omega / S=0.0$} & $b_{11}$ & 0 & 0.353 & 0 & 0 & 0.201 & 0.30 \\
\cline { 2 - 8 } & $b_{22}$ & 0 & -0.252 & 0 & 0 & -0.147 & -0.26 \\
\cline { 2 - 8 } & $b_{12}$ & -0.212 & -0.212 & -0.489 & -0.247 & -0.150 & -0.15 \\
\cline { 2 - 8 } & $S K / \varepsilon$ & 4.71 & 4.71 & 11.70 & 5.49 & 6.08 & - \\
\hline \hline \multirow{5}{*}{$\Omega / S=0.25$} & $b_{12}$ & 0 & 0.050 & 0 & 0 & - & 0.06 \\
\cline { 2 - 8 } & $b_{22}$ & 0 & 0.050 & 0 & 0 & - & 0.05 \\
\cline { 2 - 8 } & $b_{12}$ & -0.212 & -0.212 & -0.489 & -0.218 & - & -0.35 \\
\cline { 2 - 8 } & $S K / \varepsilon$ & 4.71 & 4.71 & 11.70 & 4.84 & - & - \\
\hline \hline \multirow{5}{*}{$\Omega / S=0.5$} & $\bar{b}_{11}$ & 0 & -0.252 & 0 & 0 & - & -0.26 \\
\cline { 2 - 8 } & $b_{22}$ & 0 & 0.353 & 0 & 0 & - & 0.25 \\
\cline { 2 - 8 } & $b_{12}$ & -0.212 & -0.212 & -0.489 & -0.194 & - & -0.10 \\
\cline { 2 - 8 } & $S K / \varepsilon$ & 4.71 & 4.71 & 11.70 & 4.30 & - & - \\
\hline
\end{tabular}

Table 1. Equilibrium results for homogeneous turbulent shear flow in a rotating frame. Comparison of the predictions of the two-equation models with the Large-Eddy Simulations of Bardina, Ferziger, and Reynolds (1983) and the experiments of Tavoularis and Corrsin (1981).

\begin{tabular}{|c|c|c|c|c|c|c|c|c|}
\hline & $\begin{array}{c}\text { Equilibrium } \\
\text { Values }\end{array}$ & $\begin{array}{c}\text { LRR } \\
\text { Model }\end{array}$ & $\begin{array}{c}\text { RK } \\
\text { Model }\end{array}$ & $\begin{array}{c}\text { SL } \\
\text { Model }\end{array}$ & $\begin{array}{c}\text { FLT } \\
\text { Model }\end{array}$ & $\begin{array}{c}\text { RNG } \\
\text { Model }\end{array}$ & Experiments & $\begin{array}{c}\text { Large-Eddy } \\
\text { Simulations }\end{array}$ \\
\hline \multirow{3}{*}{$\mathrm{n} / S=0$} & $b_{11}$ & 0.191 & 0.242 & 0.114 & 0.214 & 0.533 & 0.201 & 0.30 \\
\cline { 2 - 9 } & $b_{22}$ & -0.095 & -0.121 & -0.116 & -0.148 & -0.267 & -0.147 & -0.26 \\
\cline { 2 - 9 } & $b_{12}$ & -0.184 & -0.169 & -0.122 & -0.144 & 0 & -0.150 & -0.15 \\
\cline { 2 - 9 } & $S K / \varepsilon$ & 5.42 & 3.71 & 6.93 & 7.28 & $\infty$ & 6.08 & - \\
\hline \hline \multirow{3}{*}{$\Omega / S=0.25$} & $b_{11}$ & -0.059 & 0.06 & -0.070 & -0.134 & 0.159 & - & 0.06 \\
\cline { 2 - 9 } & $b_{22}$ & 0.155 & 0.06 & 0.067 & 0.200 & 0.159 & - & 0.05 \\
\cline { 2 - 9 } & $b_{12}$ & -0.208 & -0.248 & -0.153 & -0.160 & -0.449 & - & -0.35 \\
\cline { 2 - 9 } & $S K / \varepsilon$ & 4.83 & 2.53 & 5.51 & 6.54 & 12.70 & - & - \\
\hline \hline \multirow{3}{*}{$\Omega / S=0.5$} & $b_{11}$ & -0.120 & -0.121 & -0.123 & -0.151 & -0.267 & - & -0.26 \\
\cline { 2 - 9 } & $b_{22}$ & 0.160 & 0.242 & 0.120 & 0.061 & 0.533 & - & 0.25 \\
\cline { 2 - 9 } & $b_{12}$ & 0 & -0.169 & 0 & 0 & 0 & - & -0.10 \\
\cline { 2 - 9 } & $S K / \varepsilon$ & $\infty$ & 3.71 & $\infty$ & $\infty$ & $\infty$ & - & - \\
\hline
\end{tabular}

Table 2. Equilibrium results for homogeneous turbulent shear flow in a rotating frame. Comparison of the predictions of the second-order closure models with the Large-Eddy Simulations of Bardina, Ferziger, and Reynolds (1983) and the experiment of Tavoularis and Corrsin (1981). (LRR $\equiv$ Launder, Reece and Rodi, RK $\equiv$ Rotta-Kolmogorov, SL $\equiv$ Shih-Lumley, FLT $\equiv$ Fu, Launder and Tselepidakis). 


\section{List of Figures}

Figure 1. Homogeneous turbulent shear flow in a rotating frame.

Figure 2. Time evolution of the turbulent kinetic energy in rotating shear flow. Comparison of the predictions of the two-equation models with the Large-Eddy Simulations of Bardina, et al. (1983): (a) $\Omega / S=0$, (b) $\Omega / S=0.25$, and (c) $\Omega / S=0.5$.

Figure 3. Equilibrium diagram for the two-equation turbulence models in rotating shear flow.

Figure 4. Time evolution of the turbulent kinetic energy in rotating shear flow. Comparison of the predictions of the second-order closure models with the Large-Eddy Simulations of Bardina, et al. (1983): (a) $\Omega / S=0$, (b) $\Omega / S=0.25$, and (c) $\Omega / S=0.5$.

Figure 5. Equilibrium diagram for the second-order closure models in rotating shear flow. 
LES O

Standard and Nonlinear $K-\varepsilon$ Model

RNG $K-\varepsilon$ Model -..-

Bardina $K-\varepsilon$ Model ...

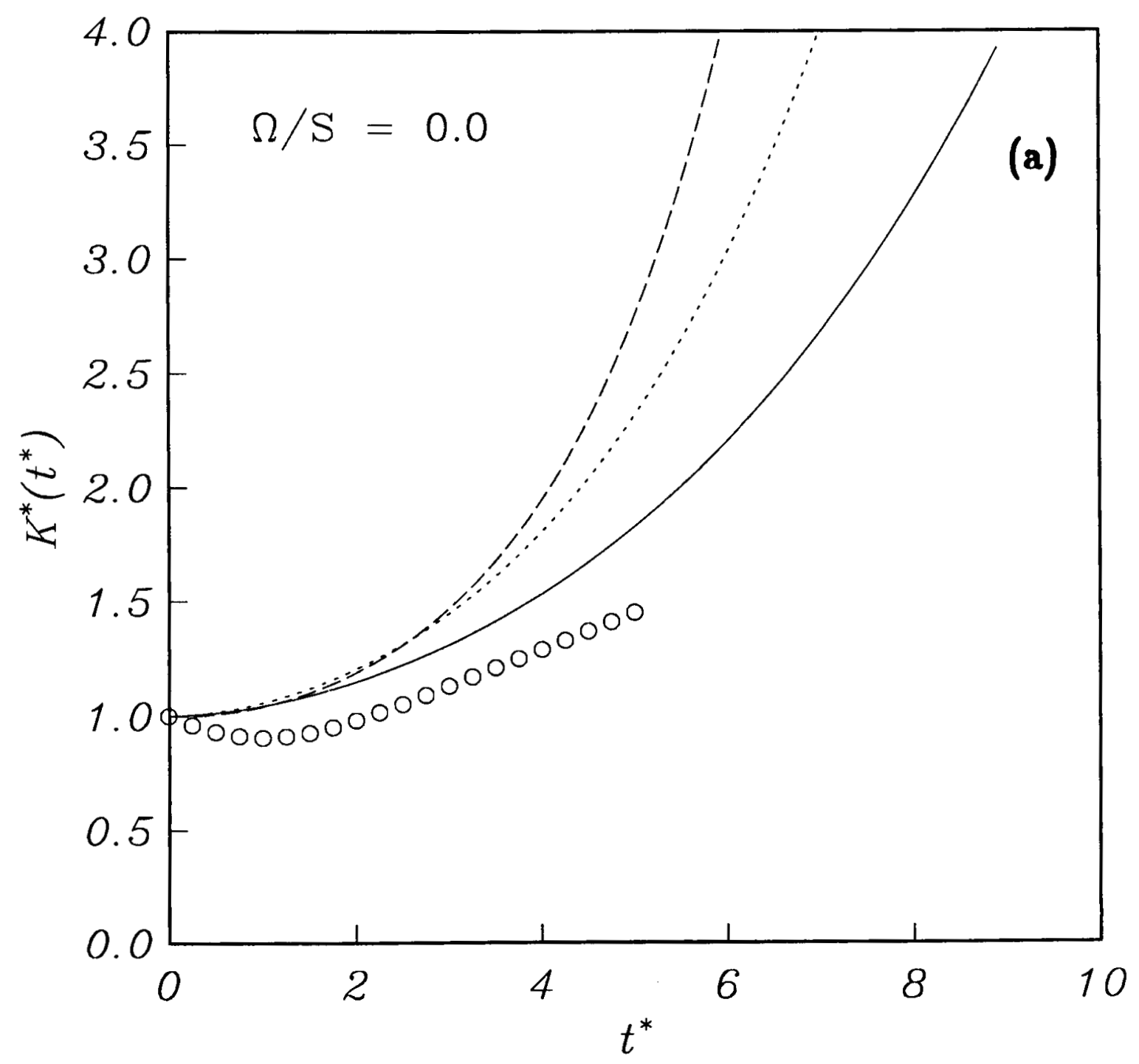

Figure 2. Time evolution of the turbulent kinetic energy in rotating shear flow. Comparison of the predictions of the two-equation models with the Large-Eddy Simulations of Bardina, et al. (1983): (a) $\Omega / S=0$, (b) $\Omega / S=0.25$, and (c) $\Omega / S=0.5$. 


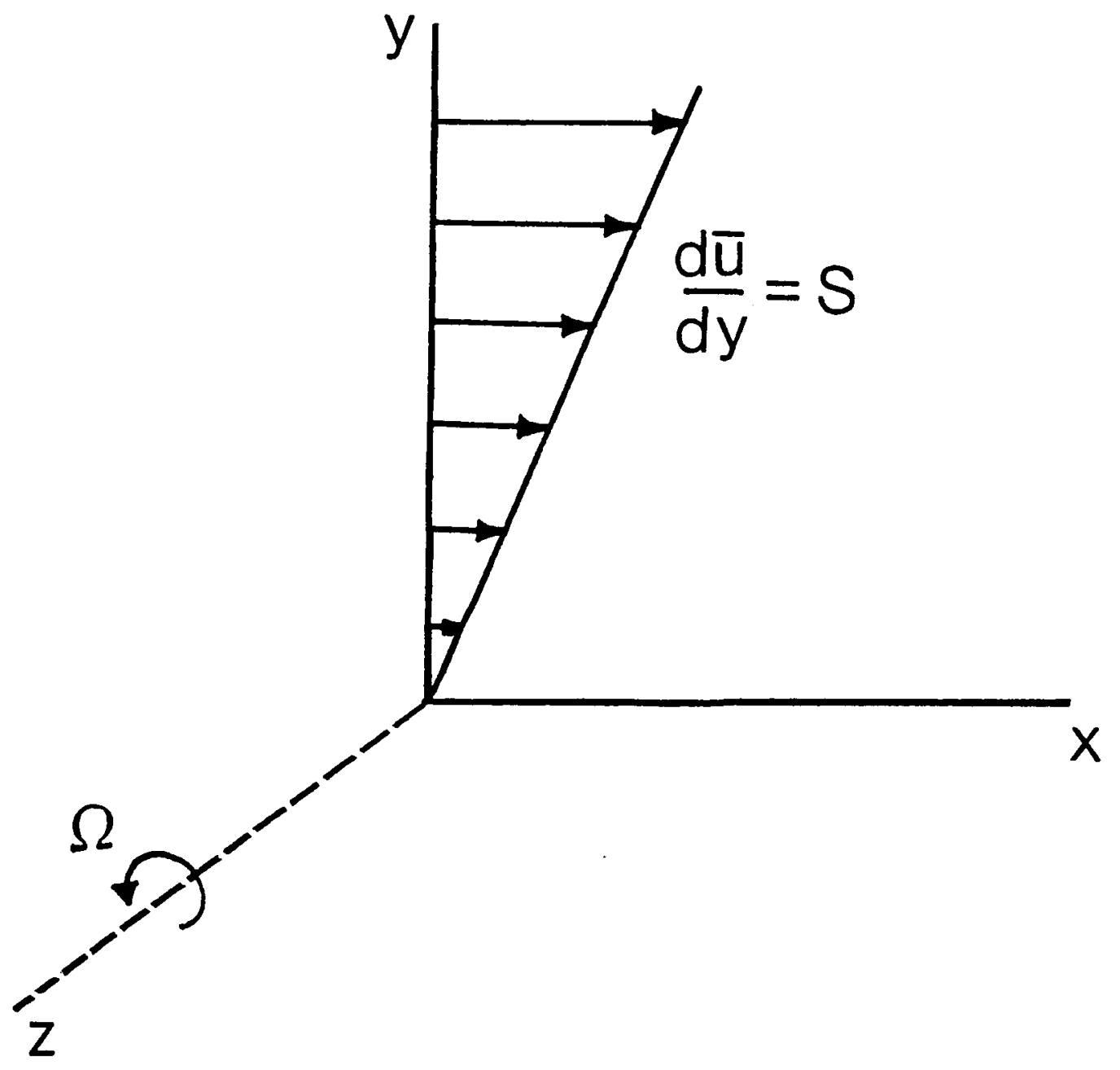

Figure 1. Homogeneous turbulent shear flow in a rotating frame. 


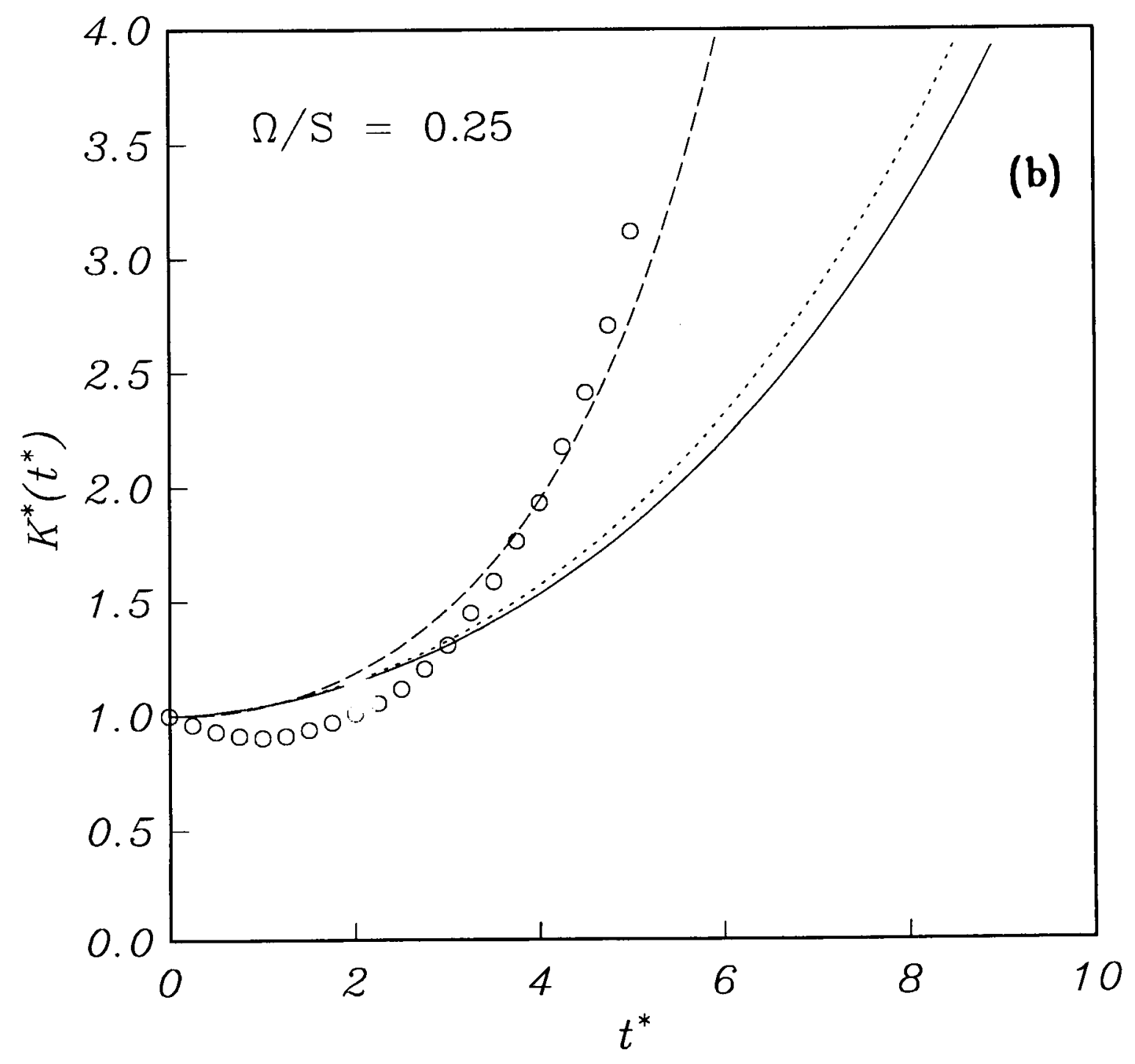

Figure 2(b). 


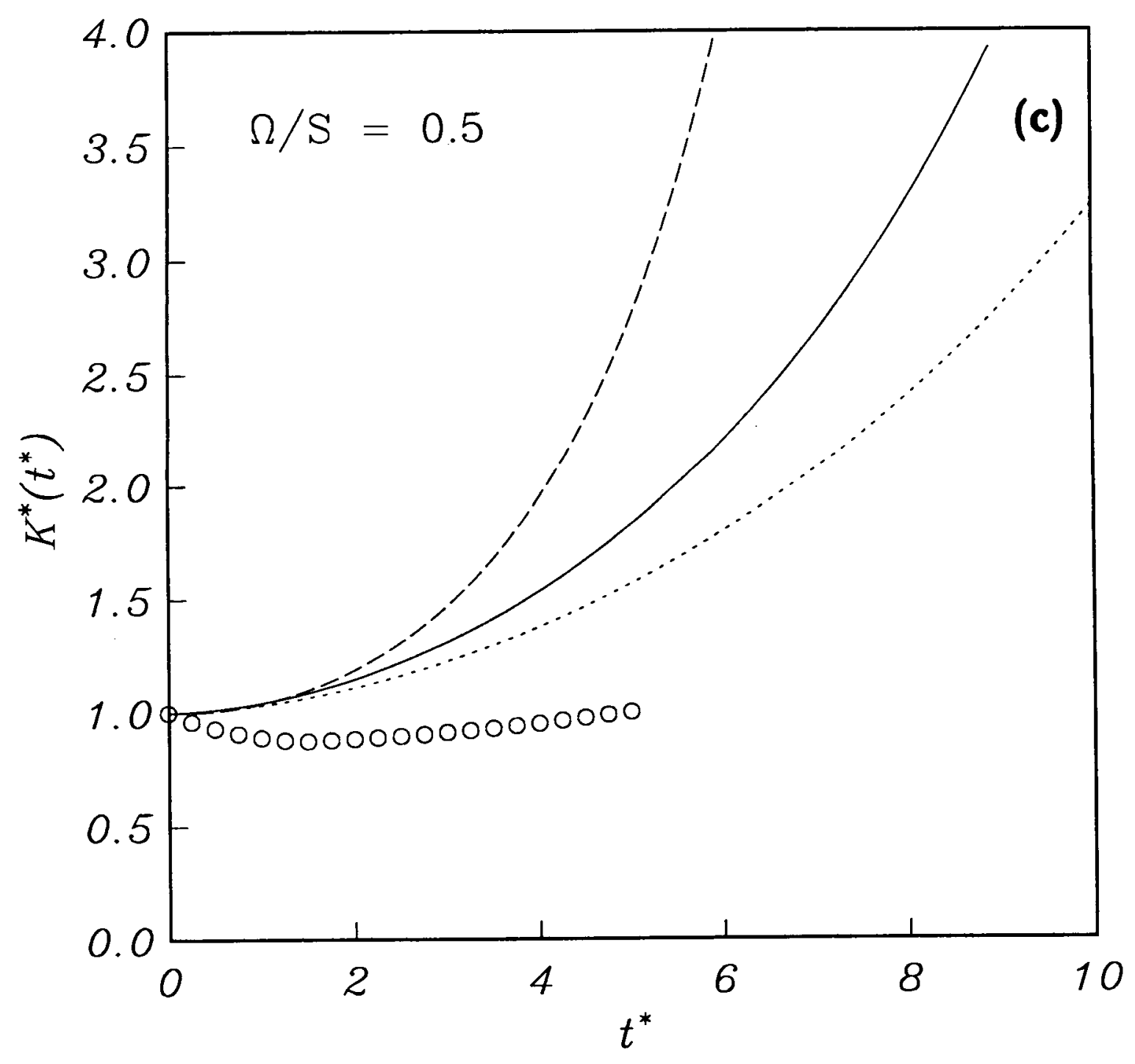

Figure 2(c). 

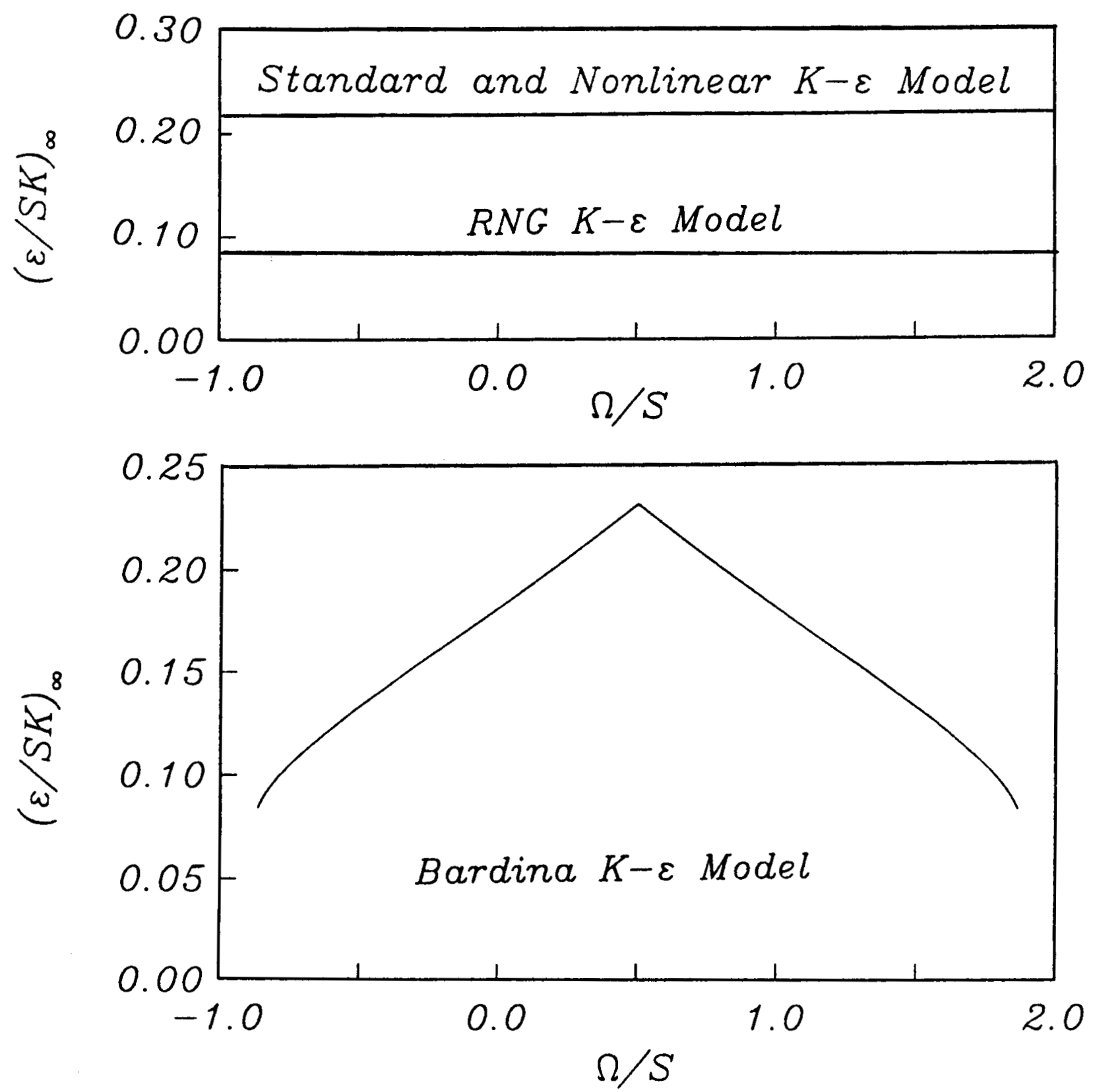

Figure 3. Equilibrium diagram for the two-equation turbulence models in rotating shear flow. 


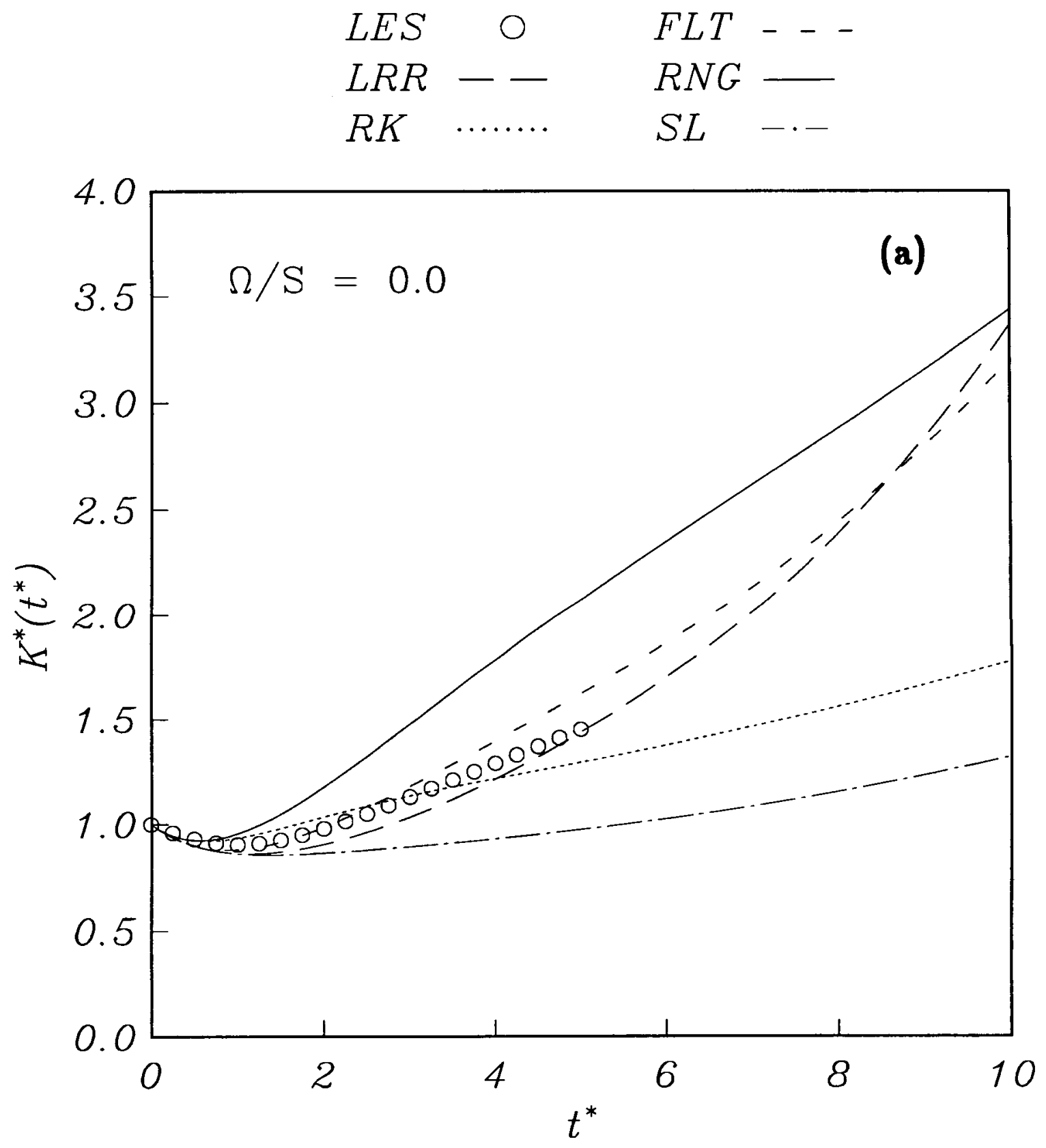

Figure 4. Time evolution of the turbulent kinetic energy in rotating shear flow. Comparison of the predictions of the second-order closure models with the Large-Eddy Simulations of Bardina, et al. (1983): (a) $\Omega / S=0$, (b) $\Omega / S=0.25$, and (c) $\Omega / S=0.5$. 


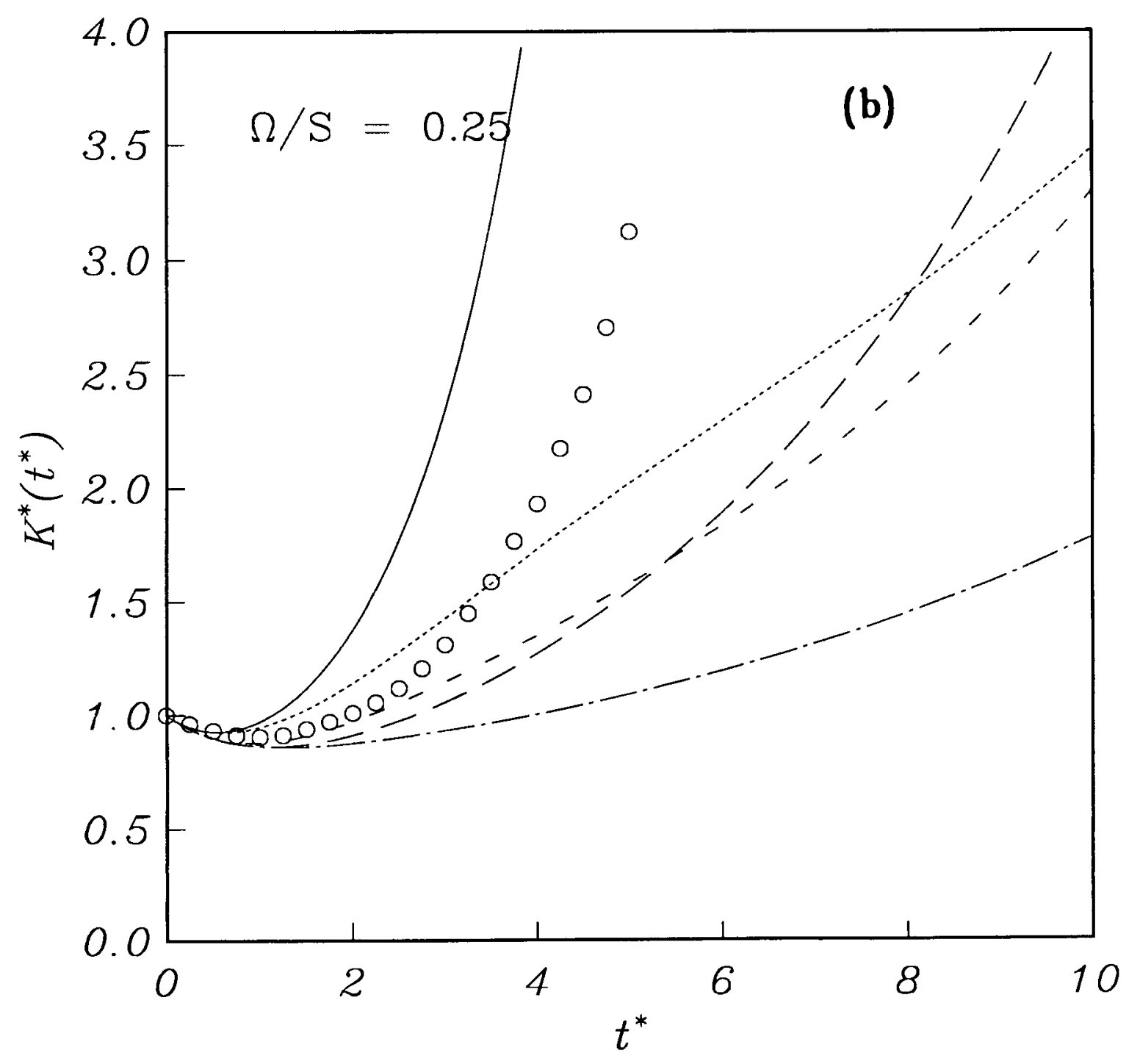

Figure 4(b). 


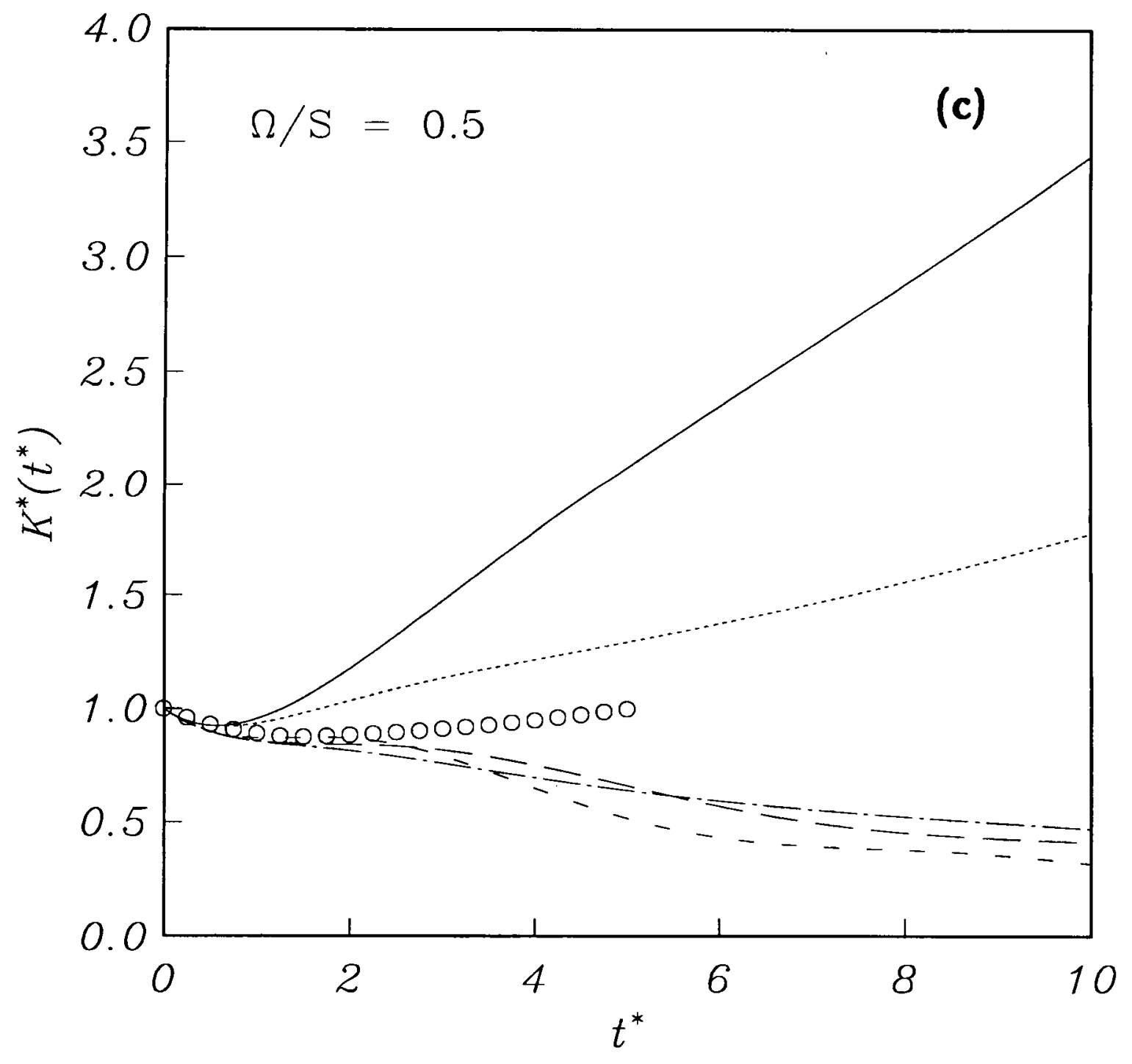

Figure 4(c). 


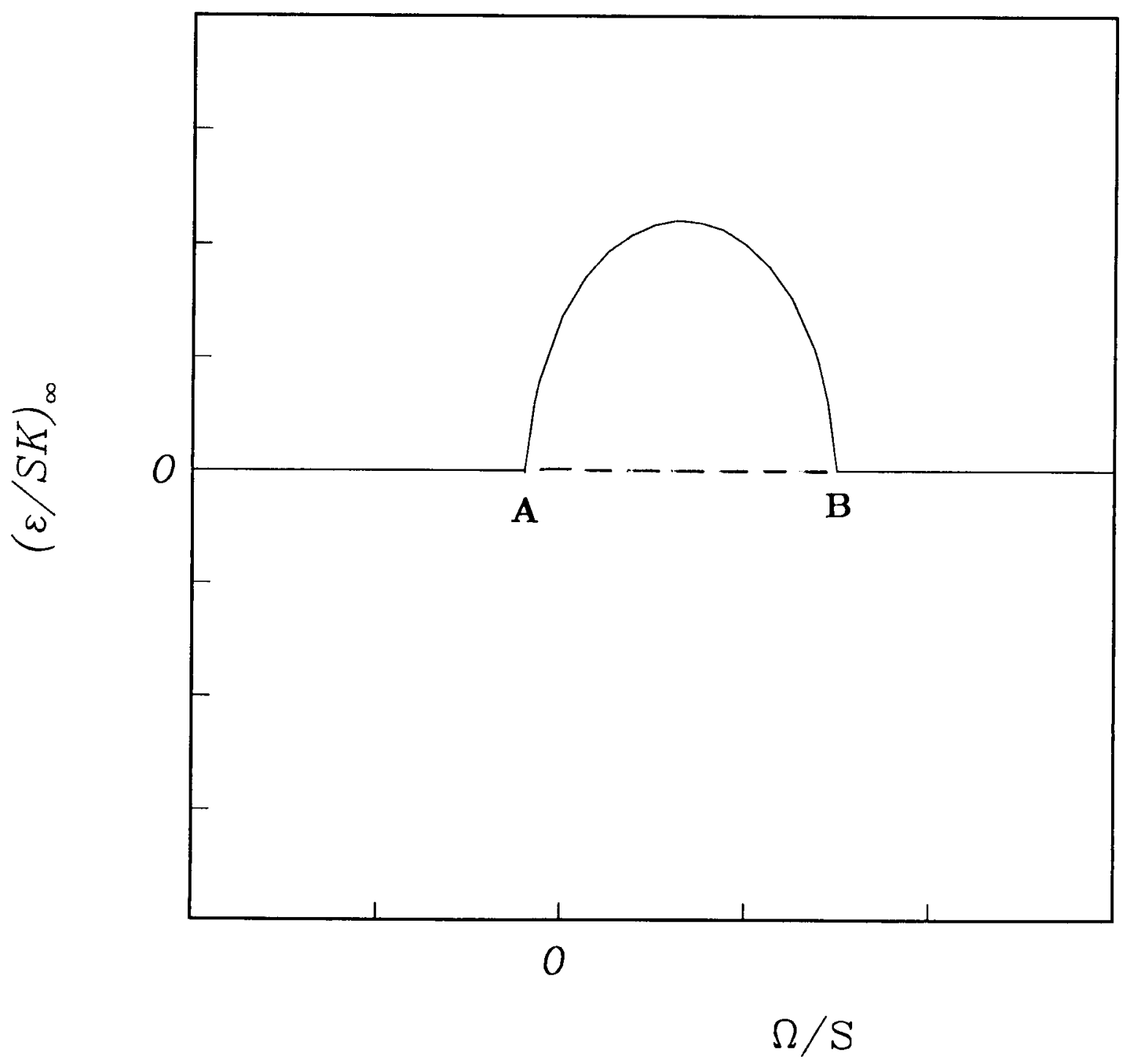

Figure 5. Equilibrium diagram for the second-order closure models in rotating shear flow. 
1. Report No.

2. Government Accession No.

3. Recipient's Catalog No.

NASA CR-181864

ICASE Report No. $89-43$

4. Title and Subtitle

A CRITICAL COMPARISON OF TURBULENCE MODELS

FOR HOMOGENEOUS SHEAR FLOWS IN A ROTATING FRAME

7. Author(s)

C. G. Speziale

T. B. Gatski

N. Mac Giolla Mhuiris

9. Performing Organization Name and Address

Institute for Computer Applications in Science and Englneering

Ma11 Stop 132C, NASA Langley Research Center

Hampton, VA 23665-5225

12. Sponsoring Agency Name and Address

National Aeronautics and Space Administration

Langley Research Center

Hampton, VA 23665-5225

5. Report Date

June 1989

6. Performing Organization Code

8. Performing Organization Report No.

$89-43$

10. Work Unit No.

505-90-21-01

11. Contract or Grant No.

NAS $1-18605$

13. Type of Report and Period Covered Contractor Report

14. Sponsoring Agency Code

15. Supplementary Notes

Langley Technical Monitor:

Richard W. Barnwel1

Seventh Symposium on Turbulent

Shear Flows

Final Report

16. Abstract

A variety of turbulence mode1s, including five second-order closures and four two equation models, are tested for the problem of homogeneous turbulent shear flow in a rotating frame. The model predictions for the time evolution of the turbulent kinetic energy and dissipation rate, as well as those for the equilibrium states, are compared with the results of physical and numerical experiments. Most of the two-equation models predict the same results for all rotation rates in which there is an exponential time growth of the turbulent kinetic energy and dissipation rate. The second-order closures are qualitatively superior since, consistent with physical and numerical experiments, they only predict this type of unstable flow for intermediate rotation rates in the range $-0.1 \leqslant \Omega / \mathrm{S}<0.6$. For rotation rates outside this range, there is an exchange of stabilities with a solution whose kinetic energy and dissipation rate decay with time. Although the secondorder closures are superior to the two-equation models, there are still problems with the quantitative accuracy of their predictions.

17. Key Words (Suggested by Author(s))

turbulent shear flow; rotating

frames; $k-\varepsilon$ models; second-order closure models
18. Distribution Statement

34 - Fluid Mechanics \& Heat Transfer

Unclassified - Unlimited
19. Security Clossif. tof this report)

Unclassified
20. Security Classif. Iof this pagel
Unclassified

\begin{tabular}{|l|r}
\hline 21. No. of pages & 22. Price \\
23 & AO 3
\end{tabular}

